\title{
Multimodality Treatment for Patients with Node-Positive Prostate Cancer: the Role of Radiation Therapy
}

\author{
Satoru Ochiai $^{1 *}$, Yoshihito Nomoto ${ }^{2}$, Shigeki Kobayashi ${ }^{2}$, Yasufumi Yamashita ${ }^{1}$, \\ Yui Watanabe ${ }^{2}$, Yutaka Toyomasu ${ }^{2}$, Tomoko Kawamura ${ }^{2}$, Akinori Takada ${ }^{2}$, \\ Noriko II ${ }^{2}$, Hajime Sakuma ${ }^{2}$
}

\begin{abstract}
Prostate cancer is the secondary most frequently diagnosed cancer in the world. Although numerous prospective randomized trial have been conducted to guide the management of patients with localized or locally advanced prostate cancer, few clinical trials targeting node-positive prostate cancer have been reported. Therefore, there are still controversies in the optimal management of node-positive prostate cancer. Recently, efficacy of multimodality treatment, including radiation therapy (RT), for such patients has been reported in several articles. The results indicate potential benefit of RT both in adjuvant therapy after prostatectomy and in definitive therapy for node-positive prostate cancer. The aim in this article was to summarize the current evidence for RT and evaluate the role in multimodality treatment for patients with node-positive prostate cancer.
\end{abstract}

Keywords: Node-positive prostate cancer - radiation therapy - androgen deprivation therapy - radical prostatectomy

Asian Pac J Cancer Prev, 17 (4), 1625-1630

\section{Introduction}

Prostate cancer is the second most frequently diagnosed cancer in men and 1.1 million new cases are estimated to have occurred in the world. It is estimated that prostate cancer contributes to more than three hundreds of thousands deaths each year (Torre et al., 2015). Although the incidence of clinically node-positive prostate cancer without distant metastasis is unclear, it is estimated approximately $10 \%$ in some reports (Créhange et al., 2012; , Baker et al., 2015). However, the incidence of node-involvement is underestimated because patients sometimes diagnosed without pelvic lymph nodes dissection (PLND) (Swanson et al., 2006; Briganti et al., 2008; Briganti et al., 2009; Créhange et al., 2012).

Although numerous randomized trials have been conducted to guide the management of patients with localized prostate cancer, few clinical trials target at patients with node-positive prostate cancer have been reported. Therefore, there is controversy in the appropriate management for patients with node-positive prostate cancer. Recently, the benefit of multimodality treatment, including radiation therapy (RT), has been described in several reports.

The purpose of this review is summarizing the results of existing literature on use of RT for node-positive prostate cancer. In this article, the role of RT in adjuvant treatment after radical prostatectomy (RP) and, in initial treatment for node-positive prostate cancer was reviewed separately.

Adjuvant treatment for pathologically node-positive patients after RP androgen deprivation therapy (ADT)

Adjuvant ADT is considered to be the standard treatment for patients with pathologically node-positive prostate cancer after RP and PLND.

In Eastern Cooperative Oncology Group Study EST 3886, 98 patients who underwent radical prostatectomy and pelvic lymphadenectomy and who found to have nodal metastases between 1988 and 1993 were randomly assigned to receive immediate ADT or to be followed until disease progression (Messing et al. 1999; Messing et al., 2006). At median follow-up of 11.9 years, men assigned immediate ADT had a significant improvement in overall survival (OS) (Hazard ratio [HR] 1.84, 95\% confidence interval [CI]: 1.01-3.35, p=0.04), cancer-specific survival (CSS) (HR 4.09, 95\% CI: 1.76-9.49, p=0.0004) and progression-free survival (PFS) (HR 3.42, 95\% CI: 1.96$5.98, \mathrm{p}<0.0001)$ compared those assigned to be followed until disease progression (Messing et al., 2006).

Iversen et al. (2004) reported an exploratory subgroup analysis assessing the extent to which the overall benefit in the Early Prostate Cancer Program is dependent on lymph node status at randomization. In the study, 
8,113 patients with localized / locally advanced disease received bicalutamide or placebo once daily, plus standard care. Compared with standard care alone, bicalutamide significantly reduced the risk of objective progression, irrespective of lymph node status, with the most pronounced reduction in patients with node-positive disease (HR 0.29, 95\% CI 0.15-0.56) compared with those with N0 (HR 0.59, $95 \%$ CI: 0.48-0.73) and Nx (HR $0.60,95 \%$ CI: 0.50-0.72) disease. The largest decrease in risk of PSA doubling with bicalutamide was observed in patients with node-positive disease (HR 0.16, 95\% CI: $0.09-0.29)$, with significantly reduced risks seen in N0 (HR 0.45, 95\% CI: $0.40-0.51$ ) and Nx (HR 0.38, 95\% CI: 0.33-0.44) disease.

Kunath et al. (2013) conducted a systematic review to determine the benefits of early (at the time of local therapy) versus deferred (at the time of clinical disease progression) ADT for patients with node-positive prostate cancer after local therapy. Three hundred ninety eight patients of four studies were included in the analysis. Early ADT lead to a significant decrease in overall mortality (OM) (HR 0.62, 95\% CI: 0.46-0.84), cancer-specific mortality (CSM) (HR $0.34,95 \%$ CI: $0.18-0.64)$, and clinical progression at 3 or 9 years (Risk ratios [RR] 0.29, 95\% CI: $0.16-0.52$ at 3 years and RR $0.49,95 \%$ CI: $0.36-0.67$ at 9 years).

\section{Addition of RT to adjuvant ADT}

Da Pozzo et al. retrospectively evaluated the role of adjuvant RT in node-positive patients after RP(Da Pozzo et al., 2009). A total of 250 consecutive patients with pathologic lymph node invasion were included analysis. One hundred twenty nine patients $(51.6 \%)$ were treated with combination of RT and ADT, while 121 patients $(48.4 \%)$ received adjuvant ADT alone. In multivariable Cox regression models, adjuvant RT was shown to be the independent predictor of biochemical recurrence-free survival (RFS) $(\mathrm{p}=0.002)$ as well as CSS $(\mathrm{p}=0.009)$.

Briganti et al retrospectively assessed the impact of combination adjuvant ADT and RT on survival of patients with prostate cancer and histologically documented pathologically lymph node metastases (Briganti et al., 2011). In the study, patients treated with adjuvant ADT + RT and patients treated with adjuvant ADT alone were matched for age at surgery, pathologic $\mathrm{T}$ stage and Gleason score, number of nodes removed, surgical margin status, and length of follow-up. One hundred seventeen pT2-4, pN1 patients of $171(68.4 \%)$ treated with adjuvant ADT + RT were compared with 247 pT2-4, pN1 patients of 532 $(46.4 \%)$ receiving adjuvant ADT alone. Patients treated adjuvant RT + ADT had significantly higher CSS and OS rates compared with patients with ADT. The 5, 8 and 10year CSS rate was $95 \%, 91 \%$ and $86 \%$, respectively, for patients who received ADT + RT versus $86 \%, 78 \%$ and $70 \%$, respectively, for patients who received ADT alone $(\mathrm{p}=0.004)$. The 5, 8 and 10-year OS rate was $90 \%, 84 \%$ and $75 \%$, respectively, for patients who received ADT + RT versus $82 \%, 65 \%$ and $55 \%$, respectively, for patients who received ADT alone ( $\mathrm{p}<0.001)$.

Abdollah et al. (2014) evaluated 1,107 patients with pN1 prostate cancer treated with RP, PLND, and adjuvant therapy between 1988 and 2010. All patients received adjuvant ADT and 35\% of patients received adjuvant RT. The 10 -year CSM-free rate was $84 \%$ in the entire cohort and $87 \%$ in patients treated with adjuvant RT plus adjuvant ADT versus $82 \%$ in patients treated with adjuvant ADT alone $(\mathrm{p}=0.08)$. At multivariate analyses, adjuvant RT status was one of the statistically significant predictors of CSM. Abdollah et al. also investigated the impact of adjuvant RT on survival of patients with pathologically node-positive prostate cancer (Abdollah et al., 2014). At multivariable analysis, adjuvant RT was associated with favorable CSM rate (HR 0.37, p<0.001). The benefit of adjuvant RT was restricted in two groups: (1) patients with positive lymph node count 2 or less, Gleason score 7 to $10, \mathrm{pT} 3 \mathrm{~b} / \mathrm{pT} 4$ stage or positive surgical margins (HR $0.30, \mathrm{p}=0.002$ ) and (2) patients with positive lymph node count of 3 to 4 (HR $0.21, p=0.02$ ). These results were also confirmed when OM was examined as an end point.

The comparison of treatment outcome between adjuvant ADT alone and combined therapy of ADT and RT is summarized in Table 1. According to the findings of these retrospective studies, it is possible that adjuvant RT plus adjuvant ADT improve the outcome of selected patients with $\mathrm{pN} 1$ prostate cancer treated with RP and PLND compared with adjuvant ADT alone. Although prospective evidence is still lacking at this time, adjuvant RT could be one of the treatment option for patients with node-positive prostate cancer after RP.

\section{Initial treatment for patients with clinically or pathologically node-positive prostate cancer}

ADT is one of the treatment options for patients with node-positive prostate cancer. In the European Organisation for the Research and Treatment of Cancer (EORTC) 30846 trial, 234 patients with lymph nodepositive (pN1-3) were randomized to immediate versus delayed endocrine treatment without treatment of the primary tumor (Schroder et al., 2004; Schroder et al., 2009). The endocrine treatment consisted of a depot luteinizing hormone-releasing hormone (LHRH) agonist and 1 month of antiandrogen treatment or surgical castration. After a median follow-up of 13 years, 193 patients $(82.5 \%)$ had died, and $59.4 \%$ of them as a result of prostate cancer (Schroder et al., 2009). The median overall survival was 6.1 years (95\%CI, 5.7-7.3) for delayed ADT group and 7.6 years $(95 \% \mathrm{CI}, 6.3-8.3)$ for immediate ADT group. Ten-year cumulative incidence of death resulting from prostate cancer was $55.6 \%$ in the delayed ADT group and $52.1 \%$ in the immediate ADT group. The treatment outcome was not statistically significantly different between the treatment arms.

Although the results of the trial indicate the potential advantage, the benefit of early treatment with ADT, compared with watch-and-wait approach, is still unclear.

\section{Role of local therapy for patients with node- positive prostate cancer}

Tward et al. (2013) evaluated the effect of RT on prostate cancer specific survival with node positive cancer 
Table 1. Summary of Comparison between Adjuvant ADT Alone and Combined Therapy of ADT and RT

\begin{tabular}{ccccc}
\hline Author & Study type & $\begin{array}{c}\text { Total number } \\
\text { of patients }\end{array}$ & Treatment & Outcome \\
\hline Briganti et al. 2011 & Two institutions, retrospective & 364 & $\begin{array}{c}\text { ADT alone } \\
\text { ADT+RT }\end{array}$ & $\begin{array}{c}8 \text {-yr CSS 78\%, OS 65\% } \\
\text { Abdollah et al. 2014 }\end{array}$ \\
& Two institutions, retrospective & 1,107 & ADT alone & ADT 84\% \\
Abdollah et al. 2014 & Two institutions, retrospective & 1,107 & ADT alone & 8-yr CSM-free 92\%, OM free 88\% \\
& & & ADT+RT & 8-yr CSM-free 86\%, OM-free 75\% \\
\hline
\end{tabular}

ADT: androgen deprivation therapy; RT: radiation therapy; yr: year; CSS: cancer-specific survival rate; OS: overall survival rate; CSM-free: cancerspecific mortality-free rate; OM-free: overall mortality-free rate

Table 2. Summary of Comparisons between ADT Alone and Combined Therapy of ADT and RT

\begin{tabular}{ccccc}
\hline Author & Study type & $\begin{array}{c}\text { Total number } \\
\text { of patients }\end{array}$ & Treatment & Outcome \\
\hline Zagars et al. 2001 & single institution, retrospective & 255 & $\begin{array}{c}\text { ADT alone } \\
\text { ADT+RT }\end{array}$ & $\begin{array}{c}\text { 5-yr OS 83\%, 10-yr OS 46\% } \\
\text { 5-yr OS 92\%, 10-yr OS 67\% }\end{array}$ \\
Lin et al. 2015 & $\begin{array}{c}\text { population based, retrospective, } \\
\text { (NCDB) }\end{array}$ & 628 & $\begin{array}{c}\text { ADT alone } \\
\text { ADT+RT }\end{array}$ & $\begin{array}{c}5 \text {-yr OS 53\% } \\
\text { 5-yr OS 72\% }\end{array}$ \\
James et al. 2015 & $\begin{array}{c}\text { multi-institutions, prospective } \\
\text { (exploratory analysis) }\end{array}$ & 155 & $\begin{array}{c}\text { not planned for RT } \\
\text { planned for RT }\end{array}$ & $\begin{array}{l}\text { 2-yr FFS 55\% } \\
\text { 2-yr FFS 85\% }\end{array}$ \\
\hline
\end{tabular}

NCDB: National Cancer Data Base; ADT: androgen deprivation therapy; RT: radiation therapy; yr: year; OS: overall survival rate; FFS: failure-free survival driven PSA rate

Table 3. Summary of Outcomes of Patients with Node-positive Prostate Cancer treated with RT

\begin{tabular}{ccccc}
\hline Author & Study type & $\begin{array}{c}\text { Total number } \\
\text { of patients }\end{array}$ & Treatment & Outcome \\
\hline Lawton et al. 1997 & multi-institutions, prospective & 173 & RT alone & 5-yr AS 50\%, 9-yr AS 38\% \\
Fonteyne et al. 2013 & single-institution, prospective & 80 & RT+ADT & 5-yr AS 72\%, 9-yr AS 62\% \\
Lilleby et al. 2015 & single-institution, retrospective & 58 & RT+ADT & 3-yr bRFS 81\%, cRFS 89\% \\
Mizowaki et al. 2015 & single-institution, retrospective & 42 & RT+ADT & 5-yr bRFS 67\%, 5-yr OS 85\% \\
\hline
\end{tabular}

RT: radiation therapy; ADT: androgen deprivation therapy; yr: year; AS: absolute survival rate; bRFS: biochemical recurrence-free survival rate; cRFS: clinical recurrence-free survival rate

in a retrospective Surveillance, Epidemiology and End Results (SEER) population based study. A total of 1,100 subjects with cT1-T4, cN1, M0 prostate adenocarcinoma diagnosed between 1988 and 2006 were included in the analysis. The 10-year CSS for men who had no definitive therapy was $50.3 \%$ and for those who had RT was $62.7 \%$, and significantly favor in men who had RT (HR 0.66, 95\% CI: 0.54-0.82, p< 0.01). On multivariate analysis, RT was the independently correlated with improved CSS (HR 0.67, 95\% CI: 0.54-0.84, p<0.01).

Rushoven et al. evaluated the impact of local therapy (RP, RT, or both) on survival outcome for patients with lymph node-positive, non-metastatic cancer (Rusthoven et al., 2014). A total of 796 clinically node-positive and 2991 pathologically nod-positive patients in SEER database were evaluated. On multivariate analysis, local therapy independently associated with improved OS and CSS in both the clinically node-positive and pathologically node-positive cohorts. Among pathologically nodepositive patients, no significant differences in survival were observed between RP versus RT and RP with or without adjuvant RT.

The findings of the study indicate that local therapy, including RT, may improve the survival outcome of patients with node-positive prostate cancer, and RT can provide similar survival benefit compared with RP.

\section{Comparison between ADT alone and ADT plus RT for node-positive prostate cancer}

Zagars et al. compared the treatment outcome for node-positive prostate cancer treated by early ablation with or without prostatic radiation (Zagars et al., 2001). Two hundred fifty five patients with lymphadenectomyproven pelvic nodal metastases were included the analysis. One hundred eighty three patients were treated with ADT alone and 72 patients were treated with combined ADT and RT. The 5 and 10-year OS rate for ADT alone group was $83 \%$ and $46 \%$, respectively. The 5 and 10 -year OS rate for combined ADT and RT group was $92 \%$ and $67 \%$, respectively. The outcome was superior in combined ADT and RT group compared with ADT alone group in the univariate and multivariate analyses.

Lin et al. (2015) compared the treatment outcome of patients with clinically node-positive prostate cancer between patients treated ADT alone and ADT + RT using National Caner Data Base (Lin et al., 2015). Of 3,540 total patients included the analysis, $32.2 \%$ were treated with ADT alone and $51.4 \%$ received ADT + RT. The all-cause mortality was compared between ADT alone and ADT + 
RT group using propensity score (PS) matching. After PS matching, 318 remained in each group. Compared with ADT alone, ADT + RT was associated with a $50 \%$ decrease risk of 5-year all-cause mortality (HR 0.50 , 95\%CI: $0.37-0.67$, two-sided $\mathrm{p}<0.001$; crude OS rate: $71.5 \%$ vs. $53.2 \%)$.

The Systemic Therapy for Advancing or Metastatic Prostate Cancer: Evaluation of Drug Efficacy: (STAMPEDE) is a randomized control trial that tests the addition of further treatments to ADT using a multi-arm, multistage design. The trial started to recruit men with either newly diagnosed metastatic, high-risk localized, or node-positive prostate cancer (Sydes et al., 2012). James et al. reported an exploratory analysis of the trial and evaluated the impact of RT on FFS of node-positive prostate cancer patients (James et al., 2015). Of 5,573 eligible patients randomized to the trial from October 5, 2005 , to May 1, 2014, 1,858 were allocated to the control arm. Of these, 721 (13\% of all randomized men) had non-metastatic prostate cancer, newly diagnosed within 6 months prior to randomization. RT had been encouraged in this group, but only mandated for NOM0 patients since November 2011. There were 177 patients with N+M0 disease randomized at least 1 year prior to the data freeze. Two-year survival in this N+M0 sub-cohort was $93 \%$ (95\% CI, 88-96\%), with $71 \%$ (95\% CI, 56-82\%) still alive after 5 years. Among node-positive prostate cancer patients, the 2-year failure-free survival (FFS) driven by PSA failure rate for planned for RT group and for not planned for RT group was $85 \%$ (95\% CI: 75-91\%) and $55 \%$ (95\% CI: 41- 67\%), respectively (adjusted HR 0.45, 95\% CI: 0.25-0.80) and the FFS outcome was favor in the planned use of RT. FFS was better among those planned for radical RT than those not planned: adjusted HR, 0.48 (95\% CI, 0.29-0.79), with 2-year FFS of $81 \%$ (95\% CI, $71-87 \%$ ) and $53 \%$ (95\% CI, 40-65\%).

Radiation Therapy Oncology Group (RTOG) 96-08 was A Phase III Trial of Total Androgen Suppression vs. Total Androgen Suppression Plus Definitive External Beam Irradiation for Pathologic Lymph Node Positive $(\mathrm{pN}+)$ Adenocarcinoma of the Prostate. Unfortunately, the trial was prematurely closed due to poor accrual.

The comparison of treatment outcome between ADT alone and combined therapy of ADT and RT is summarized in Table 2. Although prospective randomized evidence is still lacking, these findings indicate the significant survival benefit of combined RT and ADT compared with ADT alone for the treatment in patients with node-positive prostate cancer. The combined therapy of RT and ADT could be considered as a treatment option for such patients.

\section{Outcomes of node-positive prostate cancer treated with RT}

RTOG 85-31 was prospective randomized trial of standard external-beam irradiation plus immediate androgen suppression versus external beam irradiation alone for patients with locally advanced prostate cancer (Lawton et al., 1997). One hundred seventy-three patients in the trial had histologically involved lymph nodes. Lawton et al. reported the subset analysis of patients
Of 173 patients, 98 patients received RT plus immediate ADT with LHRH agonist, whereas 75 patients received RT alone with hormonal manipulation instituted at the time of relapse. With a median follow-up of 6.5 years for all patients and 9.5 years for living patients, estimated PFS with PSA level less than $1.5 \mathrm{ng} / \mathrm{mL}$ at 5 and 9 years was $54 \%$ and $10 \%$, respectively, for patients who received RT and immediate ADT versus $33 \%$ and $4 \%$, respectively, for patients who received RT alone $(\mathrm{p}<0.0001)$. Fiveand 9 -year absolute survival rates were $72 \%$ and $62 \%$, respectively for patients received RT and immediate ADT, and $50 \%$ and $38 \%$, respectively, for patients received RT alone $(p=0.23)$. Multivariant analysis showed that RT and immediate ADT had statistically significant impact in biochemical control $(\mathrm{p}<0.0001)$ and absolute survival $(\mathrm{p}=0.030)$. They concluded that patients with prostate cancer who had involved pelvic lymph nodes should be considered for RT + ADT rather than RT alone.

Fonteyne et al. reported a clinical outcome of hypofractionated intensity-modulated arc therapy (IMAT) for lymph node metastasized prostate cancer. Eighty patients with $\mathrm{T} 1-4 \mathrm{~N} 1 \mathrm{M} 0$ prostate cancer were treated with IMAT and 2-3 years of ADT (Fonteyne et al., 2013). A median dose of $69.3 \mathrm{~Gy}$ was prescribed in 25 fractions to the prostate. The pelvic lymph nodes received a minimal dose of $45 \mathrm{~Gy}$. A simultaneous integrated boost to $72 \mathrm{~Gy}$ and 65 Gy was delivered to the intraprostatic lesion and/ or pathologically enlarged lymph nodes, respectively. With a median follow-up of 36 months, actuarial 3-year biochemical RFS and clinical RFS was $81 \%$ and $89 \%$, respectively.

Lilleby et al. reported treatment outcomes in men with locally advanced and node-positive prostate cancer treated with combined pelvic intensity-modulated radiation therapy (IMRT) and ADT (Lilleby et al., 2015). Of the 138 patients included the study, 58 patients had nodepositive prostate cancer. All patients started neo-adjuvant ADT 6 months prior to IMRT, and ADT was continued to a maximum of 2.5 years in some patients with nodepositive and very-high risk profile. With a median followup of 4.9 years, the 5-year biochemical FFS and 5-year RFS were $71.4 \%$ and $76.2 \%$, respectively, for the entire cohort. The 5-year CSS and 5-year OS were $94.5 \%$ and $89.0 \%$. There were no statistically significant difference in 5-year biochemical FFS ( $\mathrm{p}=0.08)$, RFS $(\mathrm{p}=0.07)$ and CSS $(\mathrm{p}=0.66)$ between men with node-positive prostate cancer and those without nodal involvement. The 5-year OS was favor in men with node-positive prostate cancer compared with those without nodal involvement $(96.5 \%$ vs. $78.3 \%$, $\mathrm{p}=0.03$ ). In the multivariate analysis, high Gleason sum (910) were shown to have a strong independent prognostic impact on BFFS, RFS and OS ( $\mathrm{p}=0.001,<0.001$ and 0.005 , respectively). The duration of ADT (28 month or more) showed a significant independent association with improved CSS $(\mathrm{p}=0.02)$ and OS $(\mathrm{p}=0.001)$. On the other hand, lymph node involvement was not associated with survival endpoints.

Mizowaki et al. reported the outcome of high-dose whole pelvic IMRT with simultaneous integrated boost (SIB-IMRT) in patients with pelvic lymph node-positive prostate cancer (Mizowaki et al., 2015). Forty two patients 
with T2a-4N1M0 prostate cancer were definitively treated by SIB-IMRT. SIB-IMRT was designed to simultaneously deliver $78 \mathrm{~Gy}, 66.3 \mathrm{~Gy}$, and $58.5 \mathrm{~Gy}$ in 39 fractions to the prostate plus seminal vesicles, metastatic lymph nodes, and pelvic lymph node region, respectively. Adjuvant ADT was given in 41 patients except for one who developed severe adverse events during neo-adjuvant ADT. With a median follow-up pf 53 months, 5-year biochemical RFS was $67.4 \%$ (95\% CI: 48.0-81.0\%). 5-year OS and CSS rates at were $85.4 \%$ (95\% CI: $68.1-93.7 \%)$ and $91.8 \%$ (95\% CI: 76.5-97.3\%), respectively.

The summary of outcomes of patient with nodepositive prostate cancer treated with RT is shown in Table 3. According to these findings, RT with long-term ADT seems to provide significant benefit in patients with nodepositive prostate cancer. Recent advance of irradiation technique, such as IMRT and IMAT, allows for higherdose irradiation to targets, while sparing adjacent organ at risk, such as bowel and rectum. It could improve the treatment outcome and further investigation is warranted.

\section{Neoadjuvant chemotherapy}

GETUG 12 is a phase 3 randomised controlled trial which assesses the effects of combine docetaxel and estramustine on relapse in patients with high risk localised prostate cancer (Fizazi et al., 2015). In the trial, patients with treatment-naive prostate cancer and at least one risk factor (ie, stage T3-T4 disease, Gleason score of $>8$, prostate-specific antigen concentration $>20 \mathrm{ng} / \mathrm{mL}$, or pathological node-positive) were enrolled. All patients underwent a staging pelvic lymph node dissection. Patients were randomly assigned (1:1) to either ADT plus 4 cycles of docetaxel and estramustine, or ADT only. Local treatment was administered at 3 months. Two hundred and seven patients were assigned to ADT plus docetaxel and estramustine group and 206 patients to ADT only group. With a median follow-up of 8.8 years, 8-year RFS was $62 \%$ (95\% CI: 55-69\%) in the ADT plus docetaxel and estramustine group versus 50\% (95\% CI: 44-57\%) in the ADT only group (adjusted HR 0.71, $95 \%$ CI: 0.54-0.94, $\mathrm{p}=0.017)$. Of the patients who were treated with RT and had data available, $31(21 \%)$ of 151 in the ADT plus docetaxel and estramustine group versus 26 (18\%) of 143 in the ADT only group reported a grade 2 or higher long-term side effect $(\mathrm{p}=0.61)$.

Recently, Blanchard et al. reported a secondary analysis of the GEUG 12 trial (Blanchard et al., 2015). In the analysis, they evaluated the role of pelvic elective nodal irradiation (ENI). Of 413 patients included in the study, 358 patients were treated using primary RT. A total of 208 patients received pelvic RT and 150 prostate-only RT. In the trial, pathologically node-positive patients were more frequently received pelvic RT than pathologically node-negative patients $(\mathrm{p}<0.0001)$. In multivariate analysis, biochemical PFS was negatively impacted by $\mathrm{pN}$ stage (HR 2.52, 95\% CI: 1.78-3.54, p<0.0001), Gleason score 8 or high (HR 1.41, 95\% CI: $1.03-1.93, \mathrm{p}=0.033$ ) and PSA higher than $20 \mathrm{ng} / \mathrm{mL}$ (HR 1.41, 95\% CI: 1.02$1.96, \mathrm{p}=0.038$ ), and positively impacted by the use of chemotherapy (HR 0.66, 95\% CI: 0.48-0.9, p=0.009).
On the other hand, there was no association between biochemical PFS and use of pelvic ENI in multivariate analysis (HR 1.10, 95\% CI: 0.78-1.55, p=0.60). Pelvic ENI was not associated with increased acute or late patient reported toxicity.

Although it is unclear if the subgroup of patients with node-positive disease benefited, the treatment strategy seems to be promising. The results of the trial also indicate that RT, including pelvic ENI, can be given safely after neoadjuvant chemotherapy. The benefit of pelvic ENI on biochemical PFS was not shown in the exploratory analysis. The optimal target volume in RT for nodepositive prostate cancer is still controversial

In summary, we have summarized the role of RT in the multimodality treatment for node-positive prostate cancer. Although prospective data is still limited, the benefit of RT for the patients has been shown in numerous studies, and RT should be considered in the treatment for patients with node-positive prostate cancer. There are many unsolved issues, such as optimal dose-fractionation and target volume in RT, and further studies are needed to provide the optimal treatment.

\section{References}

Abdollah F, Karnes RJ, Suardi N, et al (2014). Predicting survival of patients with node-positive prostate cancer following multimodal treatment. Eur Urol, 65, 554-62.

Abdollah F, Karnes RJ, Suardi N, et al (2014). Impact of adjuvant radiotherapy on survival of patients with node-positive prostate cancer. J Clin Oncol, 32, 3939-47.

Baker BR, Mohiuddin JJ, Chen RC (2015). The role of radiotherapy in node-positive prostate cancer. Oncol (Williston Park), 29, 108-16.

Blanchard P, Faivre L, Lesaunier F, et al (2015). Outcome according to elective pelvic radiation therapy in patients with high-risk localized prostate cancer: a secondary analysis of the GETUG 12 Phase 3 Randomized Trial. Int J Radiat Oncol Biol Phys, [Epub ahead of print].

Briganti A, Blute ML, Eastham JH, et al (2009). Pelvic lymph node dissection in prostate cancer. Eur Urol, 55, 1251-65.

Briganti A, Capitanio U, Chun FK, et al (2008). Impact of surgical volume on the rate of lymph node metastases in patients undergoing radical prostatectomy and extended pelvic lymph node dissection for clinically localized prostate cancer. Eur Urol, 54, 794-802.

Briganti A, Karnes RJ, Da Pozzo LF, et al (2011). Combination of adjuvant hormonal and radiation therapy significantly prolongs survival of patients with pT2-4 $\mathrm{pN}+$ prostate cancer: results of a matched analysis. Eur Urol, 59, 832-40.

Créhange G, Chen CP, Hsu CC, et al (2012). Management of prostate cancer patients with lymph node involvement: a rapidly evolving paradigm. Cancer Treat Rev, 38, 956-67.

Da Pozzo LF, Cozzarini C, Briganti A, et al (2009). Longterm follow-up of patients with prostate cancer and nodal metastases treated by pelvic lymphadenectomy and radical prostatectomy: the positive impact of adjuvant radiotherapy. Eur Urol, 55, 1003-11.

Fizazi K, Faivre L, Lesaunier F, et al (2015). Androgen deprivation therapy plus docetaxel and estramustine versus androgen deprivation therapy alone for high-risk localised prostate cancer (GETUG 12): a phase 3 randomised controlled trial. Lancet Oncol, 16, 787-94.

Fonteyne V, Lumen N, Ost P, et al (2013). Hypofractionated intensity-modulated arc therapy for lymph node metastasized 


\section{Satoru Ochiai et al}

prostate cancer: early late toxicity and 3-year clinical outcome. Radiother Oncol, 109, 229-34.

Iversen P, Wirth MP, See WA, et al (2004). Is the efficacy of hormonal therapy affected by lymph node status? data from the bicalutamide (casodex) early prostate cancer program. Urol, 63, 928-33.

James ND, Spears MR, Clarke NW, et al (2015). Failure-free survival and radiotherapy in patients with newly diagnosed nonmetastatic prostate cancer: data from patients in the control arm of the STAMPEDE Trial. JAMA Oncol. [Epub ahead of print].

Kunath F, Keck B, Rücker G, et al (2013). Early versus deferred androgen suppression therapy for patients with lymph nodepositive prostatecancer after local therapy with curative intent: a systematic review. BMC Cancer, 13, 131.

Lawton CA, Winter K, Byhardt R, et al (1997). Androgen suppression plus radiation versus radiation alone for patients with $\mathrm{D} 1 \mathrm{(pN+)}$ adenocarcinoma of the prostate (results based on a national prospective randomized trial, RTOG 85-31). Radiation Therapy Oncology Group. Int J Radiat Oncol Biol Phys, 38, 931-9.

Lawton CA, Winter K, Grignon D, et al (2005). Androgen suppression plus radiation versus radiation alone for patients with stage D1/pathologic node-positive adenocarcinoma of the prostate: updated results based on national prospective randomized trial Radiation Therapy Oncology Group 85-31. J Clin Oncol, 23, 800-7.

Lilleby W, Narrang A, Tafjord G, et al (2015). Favorable outcomes in locally advanced and node positive prostate cancer patients treated with combined pelvic IMRT and androgen deprivation therapy. Radiat Oncol, 10, 232.

Lin CC, Gray PJ, Jemal A, et al (2015). Androgen deprivation with or without radiation therapy for clinically node-positive prostate cancer. J Natl Cancer Inst, 107, 119.

Messing EM, Manola J, Sarosdy M, et al (1999). Immediate hormonal therapy compared with observation after radical prostatectomy and pelvic lymphadenectomy in men with node-positive prostate cancer. $N$ Engl J Med, 341, 1781-8.

Messing EM, Manola J, Yao J, et al (2006). Immediate versus deferred androgen deprivation treatment in patients with node-positive prostate cancer after radical prostatectomy and pelvic lymphadenectomy. Lancet Oncol, 7, 472-9.

Mizowaki T, Inokuchi H, Nakamura K, et al (2015). Outcomes of high-dose whole-pelvic IMRT with simultaneous integrated boost in patients with pelvic lymph node-positive prostate Cancer. Int J Radiat Oncolo Biol Phys, 93, 188.

Rusthoven CG, Carlson JA, Waxweiler TV, et al (2014). The impact of definitive local therapy for lymph node-positive prostate cancer: a population-based study. Int J Radiat Oncol Biol Phys, 88, 1064-73.

Schroder FH, Kurth KH, Fossa SD, et al (2004). Early versus delayed endocrine treatment of $\mathrm{pN} 1-3 \mathrm{M} 0$ prostate cancer without local treatment of the primary tumor: results of european organisation for the research and treatment of Cancer 30846--a phase III study. J Urol, 172, 923-7.

Schroder FH, Kurth KH, Fossa SD, et al (2009). Early versus delayed endocrine treatment of T2-T3 pN1-3 M0 prostate cancer without local treatment of the primary tumour: final results of european organisation for the research and treatment of cancer protocol 30846 after 13 years of followup (a randomised controlled trial). Eur Urol, 55, 14-22.

Swanson GP, Thompson IM, Basler J (2006). Current status of lymph node-positive prostate cancer: incidence and predictors of outcome. Cancer, 107, 439-50.

Torre LA, Bray F, Siegel RL, et al (2015). Global cancer statistics, 2012. CA Cancer J Clin, 65, 87-108.

Sydes MR, Parmar MK, Mason MD, et al (2012). Flexible trial design in practice-stopping arms for lack-of-benefit and adding research arms mid-trial in STAMPEDE: a multi-arm multi-stage randomized controlled trial. Trials, 13, 168.

Tward JD, Kokeny KE, Shrieve DC (2013). Radiation therapy for clinically node-positive prostate adenocarcinoma is correlated with improved overall and prostate cancer-specific survival. Pract Radiat Oncol, 3, 234-40.

Zagars GK, Pollack A, von Eschenbach AC (2001). Addition of radiation therapy to androgen ablation improves outcome for subclinically node-positive prostate cancer. Urol, 58, 233-9. 